Portland State University

PDXScholar

\title{
Using the Gouy Phase Anomaly to Localize and Track Bacteria in Digital Holographic Microscopy 4D Images
}

True Gibson

Portland State University

Manuel Bedrossian

Portland State University

Eugene Serabyn

California Institute of Technology

Chris Lindensmith

Portland State University

Jay Nadeau

Portland State University, nadeau@pdx.edu

Follow this and additional works at: https://pdxscholar.library.pdx.edu/phy_fac

Part of the Physics Commons

Let us know how access to this document benefits you.

\section{Citation Details}

Gibson, T., Bedrossian, M., Serabyn, E., Lindensmith, C., \& Nadeau, J. L. (2020). Using the Gouy phase anomaly to localize and track bacteria in digital holographic microscopy 4D images. Journal of the Optical Society of America A, 38(2), A11. https://doi.org/10.1364/josaa.404004

This Post-Print is brought to you for free and open access. It has been accepted for inclusion in Physics Faculty Publications and Presentations by an authorized administrator of PDXScholar. Please contact us if we can make this document more accessible: pdxscholar@pdx.edu. 


\title{
Using the Gouy phase anomaly to localize and track bacteria in digital holographic microscopy 4D images
}

\author{
${ }^{3}$ True Gibson, ${ }^{1}$ Manuel Bedrossian, ${ }^{2}$ Eugene Serabyn, ${ }^{2}$ Chris \\ LINDENSMITH AND ${ }^{3}$ JAY L. NADEAU* \\ ${ }^{1}$ California Institute of Technology, Department of Medical Engineering, 1200 E. California Blvd., \\ Pasadena, CA 91125 USA \\ 2 Jet Propulsion Laboratory, California Institute of Technology, 4800 Oak Grove Dr., Pasadena, CA \\ 91109 \\ ${ }^{3}$ Portland State University, Department of Physics, 1719 SW 10 ${ }^{\text {th }}$ Ave, Portland, OR 97201, USA \\ *nadeau@pdx.edu
}

\begin{abstract}
Described over 100 years ago, the Gouy phase anomaly refers to the additional $\pi$ phase shift that is accumulated as a wave passes through focus. It is potentially useful in analyzing any type of phase-sensitive imaging; in light microscopy, digital holographic microscopy (DHM) provides phase information in the encoded hologram. One limitation of DHM is the weak contrast generated by many biological cells, especially unpigmented bacteria. We demonstrate here that the Gouy phase anomaly may be detected directly in the phase image using the z-derivative of the phase, allowing for precise localization of unlabeled, micron-sized bacteria. The use of dyes that increase phase contrast does not improve detectability. This approach is less computationally intensive than other procedures such as deconvolution, and is relatively insensitive to reconstruction parameters. The software is implemented in an opensource FIJI plug-in.
\end{abstract}

(C) 2019 Optical Society of America under the terms of the OSA Open Access Publishing Agreement

\section{Introduction}

In 1890, Louis Georges Gouy directly observed that a converging beam of light experiences an axial phase advance as it travels through focus. He found that this was not a property of light exclusively, but of all converging waves, including acoustic waves [1, 2]. Many theoretical analyses have since led to the verification of this axial phase shift. In 1909, Peter Debye found an exact analytical solution to the wave equation that encapsulated the phase shift observed by Gouy [3]. In 1938, Wojciech Rubinowicz explained the phenomenon by treating light using boundary diffraction theory [4]. Nearly two decades later, Edward H. Linfoot and Emil Wolf performed a full 3D calculation of the phase distribution of light from a point source using Kirchoff diffraction theory, showing that phase becomes undefined at focus [5]. Since its discovery, the Gouy phase anomaly has been observed both theoretically and experimentally in many different beam types including, but not limited to, Gaussian beams, Bessel beams, and photonic nanojets. An extensive review may be found in [6].

Most optical imaging modalities are oblivious to a phase flip due to the fact that optical detectors only measure the time-integrated electric field intensity of an incident wavefront. Some techniques benefit from the Gouy phase anomaly, however, either directly or indirectly. Interferometry is a technique where phase information is critical. For example, one type of astronomical nulling interferometer, the achromatic interfero coronagraph (AIC), exploits the Gouy phase to introduce an achromatic $\pi$ phase shift in one arm of a Michelson interferometer, 
which results in the cancellation of incident starlight, allowing very faint off-axis companions to be seen $[7,8]$.

Digital holographic microscopy (DHM) is an interferometric light microscopy technique with several possible geometries. In digital in-line holographic microscopy (DIHM), there is a single beam path that contains both the object and reference beams. Quantitative phase cannot be determined independently of amplitude without employing techniques such as phase shifting, but the Gouy phase anomaly can be observed after reconstruction of intensity image stacks for so-called "phase objects." Many unpigmented biological cells are essentially transparent at the focal plane in intensity, and thus are essentially phase objects. The use of this technique for precise z-localization of weak scatterers such as bacteria [9] has been proposed, and more recently implemented for tracking of micron-scale malaria parasite microgametes [10] and haloarchaea [11].

On the other hand, the use of off-axis DHM (OADHM) permits independent reconstruction of amplitude and quantitative phase images. Most laboratory off-axis instruments use a MachZehnder configuration in transmission geometry through a glass sample chamber[12]. In OADHM, an object is illuminated with a plane wave, and a reference beam is designed to arrive at the detector plane with the same wavefront curvature as the object wave, but at an angle $\theta$. This allows for separation in Fourier space of the zero-order or DC terms, the virtual image, and the real image, with the digital hologram representing an intensity of the sum of the absolute square of these terms:

$I_{h}=\left|E_{R}\right|^{2}+\left|E_{O}\right|^{2}+E_{R}^{*} E_{O}+E_{O}^{*} E_{R}$

where $\|^{2}$ indicates the absolute square and ${ }^{*}$ indicates the complex conjugate.

After selecting the real or virtual image in Fourier space, the selected frequencies may be reconstructed into amplitude and phase images by a deterministic reconstruction algorithm such as Fresnel transform, the convolution method, or the angular spectrum method[13].

DHM, both in-line and off-axis, has been used in a variety of biological applications. One of its advantages over brightfield microscopy is that it captures an entire sample volume in a single hologram, which is ideal for sparse samples moving in three dimensions. The frame rate is limited only by the camera speed. As mentioned, OADHM provides complete quantitative phase information, unlike traditional Zernike phase contrast, which is qualitative. The emerging field of quantitative phase imaging (QPI) relates changes in phase to physiological or pathological phenomena mostly related to the water content of cells [14]. Phase shift $\Delta \varphi$ is proportional to the optical path difference (OPD) at each pixel; OPD is the product of the sample thickness $t$ and index of refraction difference with the medium $\Delta n$ :

$\Delta \varphi=(2 \pi / \lambda) t \Delta \mathrm{n}(2)$.

Which type of image is used for tracking depends upon the cell type and imaging characteristics, particularly noise. Detecting weak scatterers, such as most prokaryotes (bacteria and archaea), is difficult in both amplitude and phase. Amplitude images are often noisier than phase images, containing a good deal of speckle noise when coherent illumination is used. This noise can be almost indistinguishable from cells whose size is near the diffraction limit. While phase images are less sensitive to speckle noise, the phase shift is exquisitely sensitive to optical aberrations, including tilt, astigmatism, chromatic aberrations, and other errors [15]. The sample must also be mounted on high-quality optical glass, rather than polymer, as polymers can show undesired internal phase features. On the other hand, phase images of weak scatterers have the advantage that they do not require unwrapping. The refractive index of most bacteria differs from water only at the percent level ( 1.38 reported for E. coli) [16], so that the phase shift observed is significantly less than $\pi$ for cells $1-5 \mu \mathrm{m}$ in thickness.

In this paper, we demonstrate a simple implementation of the use of the Gouy phase to track bacteria using pure phase images reconstructed from OADHM recordings. Holograms are reconstructed into phase images $f(x, y)$ at each $z$, with the spacing between $\mathrm{z}$ planes chosen during the reconstruction. The $z$-gradient is calculated as 
$\partial f(x, y, z) * G^{s}(z)=f^{*} \partial G^{s}(3)$,

where $G^{s}$ indicates a Gaussian weight function of scale $s$ and * indicates convolution [17], using the existing FIJI package FeatureJ. An alternative without smoothing is also provided, with the z-gradient simply calculated as the difference of frames on either side of the target $z$, with or without re-addition of the base image for added contrast:

$$
\begin{gathered}
\partial f=f(z)-f(z-2) \text { Central derivative } \\
\partial f=f(z)-f(z-2)+f(z-1) \text { Ad hoc derivative (4). }
\end{gathered}
$$

The resulting images are then used for particle tracking using standard algorithms. This represents an approach that is faster and less computationally intensive than other methods such as deconvolution, the standard in fluorescence microscopy. A FIJI plug-in for $4 \mathrm{D}$ processing is provided.

\section{Materials and Methods}

\subsection{DHM instrument and sample chambers}

A custom DHM instrument was used for this work, an off-axis common-path configuration as described previously [18] (Supporting Information Fig. S1). The effective magnification is 19.7 with a diffraction limited lateral resolution of $0.8 \mu \mathrm{m}$. The illumination wavelength was $405 \mathrm{~nm}$, supplied by a single-mode, fiber-coupled diode laser (Thorlabs S1FC405) that is collimated before the sample. The camera was an Allied Vision Prosilica GT 2450 camera (3.45 $\mu \mathrm{m} /$ pixel). Acquisition speed was 15 frames per second with all frames measuring 2048x2048 pixels. Custom sample chambers were required to provide a clean reference channel (filled with $\mathrm{dH}_{2} \mathrm{O}$ or bacterial medium) at the correct spacing from the sample. The chamber design has been described elsewhere [19].

\subsection{Bacteria and data acquisition}

Two types of test organisms, representing different sizes, swimming speeds, and phase contrast properties were used here. The first was the marine psychrophile Colwellia psychrerythraea strain 34H (gift of J. Deming, University of Washington). C. psychrerythraea was maintained in $1 / 2$ strength 2216 Marine Broth (Difco) at $4^{\circ} \mathrm{C}$. It was diluted to a concentration of $\sim 10^{7}$ cells/mL in the same medium for imaging.

The second strain tested was Escherichia coli (strain AW405), with or without staining with $4 \mu \mathrm{M}$ of a metallocorrole dye, $\mathrm{Ga}(\mathrm{tpfc})\left(\mathrm{SO}_{3} \mathrm{H}\right)_{2}$, which has a strong absorbance band at $405 \mathrm{~nm}$ (the dye was a gift of John Termini, City of Hope). For imaging, dye was added to cells in 0.9 $\% \mathrm{NaCl}$ at mid-log phase and allowed to incubate for 30-60 min. Before imaging, cells were pelleted by centrifugation at $3000 \mathrm{x}$ g and resuspended in "motility medium" (10 mM potassium phosphate, $10 \mathrm{mM} \mathrm{NaCl}, 0.1 \mathrm{mM}$ EDTA, $0.1 \mathrm{mM}$ glucose, $\mathrm{pH}$ 7.0). Staining was confirmed using widefield fluorescence microscopy; at $4 \mu \mathrm{M}$, nearly all cells were labeled as described previously[20].

\subsection{Reconstruction and processing}

Holograms were reconstructed in phase using the "DHM Utilities" plug-ins in FIJI which we have developed and published previously [21, 22]. Reconstruction is performed using the angular spectrum method, which has been shown to have advantages over other methods for quantitative phase microscopy[23]. Aberration correction by use of a reference hologram was used for all phase reconstructions; the reference hologram's negative phase is multiplied with the hologram before propagation as described in[24]. The reference hologram chosen was the median of the entire time series of holograms in each recording. Amplitude reconstruction was performed without a reference hologram but with tilt correction as described in [25]. 
Reconstruction dimensions were $365 \times 365 \times 100 \mu \mathrm{m}$, with a resolution of $2 \mu \mathrm{m}$ on the z-axis (51 planes total). Time series ranged from 51 frames or 3.4 seconds (C. psychrerythraea) to 100 frames or 6.7 seconds (both dyed and undyed E. coli). Differentiation in z was implemented as a custom plug-in given in Supporting Information. 4D reconstructions were then processed using Arivis Vision4D (arivis AG, Munich, Germany, version 3.1.1) (example pipeline shown in Supporting Information Fig. S1). Reconstructions were filtered using a sigmoid intensity filter, after which objects were identified using the "blob finder" tool. Tracks were generated from the object set and then parsed manually to ensure accuracy.

\section{Results}

\subsection{Colwellia psychrerythraea}

The challenge of DHM imaging of all micron-sized organisms at relatively low resolution, to permit high depth of field, is that cells are difficult to distinguish from noise. Holographic images contain a number of Airy rings around each scatterer, with the number of rings dependent upon the distance from the focal plane. Although these are a feature of the image reconstruction process and are not noise per se, they make automated tracking difficult, since thresholding routines detect out-of-focus Airy rings as readily (or in some cases more readily) than they detect cells. Fig. 1 shows raw holograms (Fig. 1a), median-subtracted holograms (Fig. 1b), and a single-plane amplitude reconstruction (Fig. 1c) of the marine psychrophile Colwellia psychrerythraea. Because the cells are highly motile, median subtraction of either the holograms before amplitude reconstruction [26] or of the reconstructed amplitude time series greatly improves signal to noise. However, these techniques do not pertain to phase images.
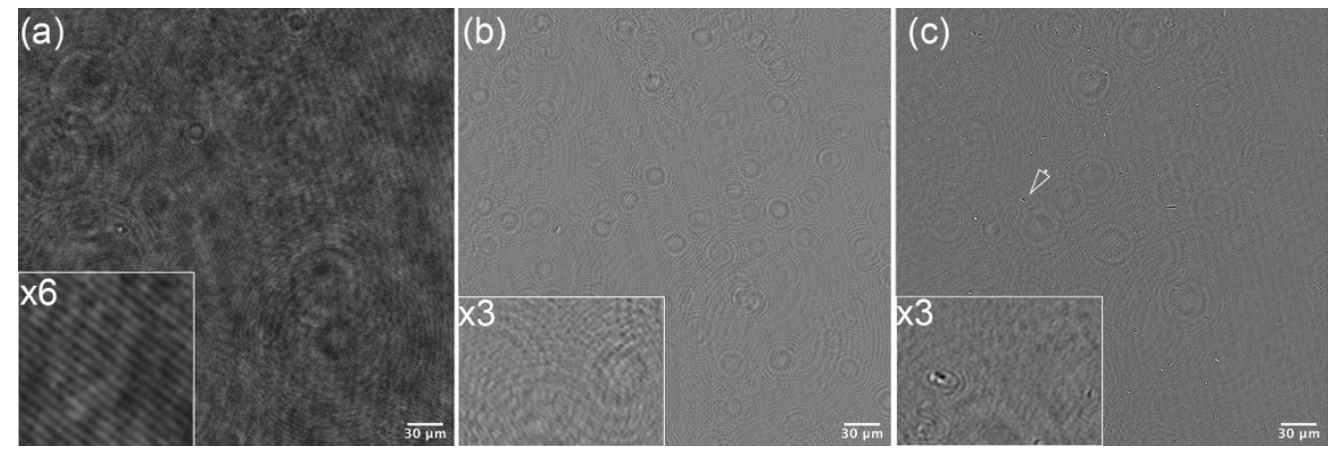

Figure 1. Holograms and amplitude reconstructions of Collwellia psychrerythraea 34H. (a) Raw hologram of the entire microscopic field of view of the instrument, with magnified inset showing fringes. (b) Median subtracted hologram showing individual cells whose patterns overlap in z. The inset is magnified 3x. (c) Reconstruction on a single focal plane in amplitude. Cells in focus (arrow) are reduced to 1-2 pixels, while cells not in focus in that plane retain large diffraction patterns. The amplified inset shows the same area in panel (b): one cell has come into focus, whereas the others have gone further out of focus in this plane.

Use of the Gouy phase anomaly makes it possible to rapidly localize each cell in a 3D volume to a its particular focal plane. The parameter choices required for implementation of the algorithm were (a) reconstruction $z$ spacing and (b) choice of derivative algorithm. For amplitude reconstructions, the choice of $z$ spacing is determined by depth of focus. However, different considerations are important for phase. The reconstruction spacing should of course be smaller than the distance over which the Gouy phase anomaly occurs, which is comparable to the depth of focus. We tested different reconstruction spacings in order to determine this empirically for our samples and to choose a $z$ spacing that optimized centroid localization while minimizing the size of the dataset. We found that choosing the $z$ step to be approximately the size of a cell $(2 \mu \mathrm{m})$ permitted the most automated analysis as it minimized false positive 
detections. This is also comparable to the depth of focus, which is given by $\lambda / \mathrm{NA}^{2}$, where NA is the numerical aperture (0.4). For $405 \mathrm{~nm}$ illumination, depth of focus is $2.5 \mu \mathrm{m}$.

Using a smaller $z$ step did decrease the size of the thresholded volume (Supporting Information Fig. S2), although values smaller than $0.5 \mu \mathrm{m}$ led to substantial spurious identification of noise as objects (Supporting Information Table S1). Using a reconstruction step size of $2 \mu \mathrm{m}$, the mean \pm standard error of the mean axial length of the Colwellia cells was $5.31 \pm .06 \mu \mathrm{m}$ (average volume, $1.68 \pm 0.05 \mu \mathrm{m}^{3}$, sphericity $0.471 \pm 0.001, n=1883$; Supporting Information Table S2). The use of derivative smoothing did not improve detection and again could lead to spurious detection of noise as cells (not shown). Thus, when using the FeatureJ plug-in, smoothing was set to " 1 " for all analysis or the simple plane-byplane method was used (the "Ad hoc" case of Eq. (4)).

Fig. 2a shows a single-plane phase reconstruction of a culture of C. psychrerythraea. It can be seen that while individual cells are clearly apparent to the eye, the out-of-focus Airy rings still present a significant barrier to thresholding and particle tracking. In the derivative image (Fig. 2b), the out-of-focus cells have disappeared from the selected plane and only the in-focus cells are visible. The difference is as much in reduction of noise as in improvement of signal, as can be appreciated from the $x y$ images (Fig $2 \mathbf{c}$,d) and $y z$ images (Fig.2 e,f) of the raw phase vs. the derivative of a single cell, and in the plot profiles shown of grayscale values along $x$ and $y$ (Fig. 2g) and through $y z$ (Fig. 2h). The full-width at half maximum of a Gaussian function fitted to the profiles in Fig. $2 \mathrm{~h}$ was consistent with the height of the objects as measured in the images.

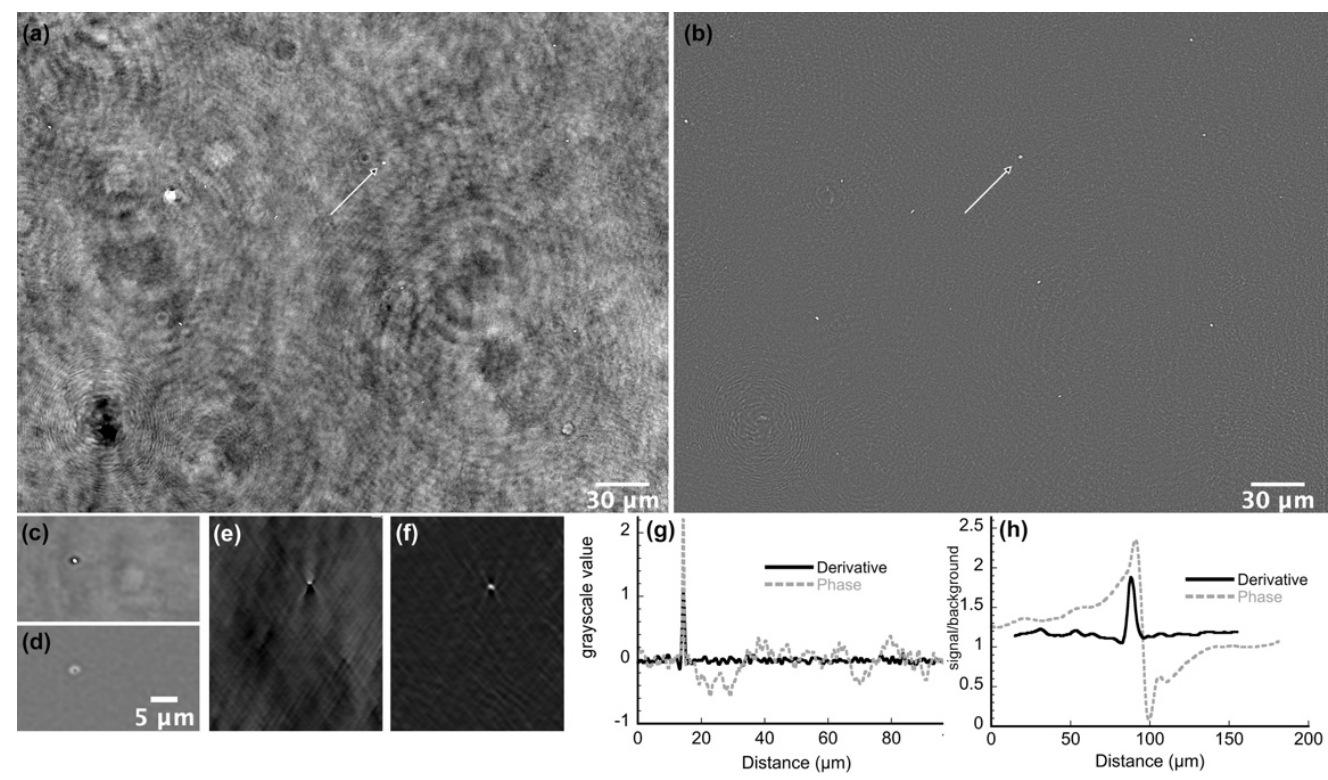

Figure 2. Phase reconstructions and derivatives of $C$. psychrerythraea. (a) Phase reconstruction on a single $\mathrm{z}$ plane, full field of view laterally (cropped vertically), with an example cell indicated by an arrow. (b) Gradient at the same plane. (c) 3x zoom of a single cel in the raw phase image, single $z$ plane. (d) Derivative image of a single cell. (e) $y z$ image through a stack of raw phase images reconstructed every $1 \mu \mathrm{m}$. (f) Derivative image of cell in the $y z$ plane. (g) Plot of signal in $x y$ for the derivative and phase images, showing a reduction of noise in the derivative image. (h) Plot of signal to noise in the yz plane.

This reduction of the Airy rings in the derivative plot allows for simple thresholding and 4D tracking of motile cells. Fig. 3a shows a 3D phase reconstruction volume before differentiation. Such a dataset cannot be effectively thresholded, and tracking must be performed manually. Fig. $3 \mathbf{b}$ shows the same dataset after differentiation, with tracks identified automatically. Each 
cell has been reduced from an extended volume spread function (Fig. 3c) to a point limited by the spatial resolution in $x, y$, and $z$ or the actual size of the object (Fig. $3 \mathbf{d})$.
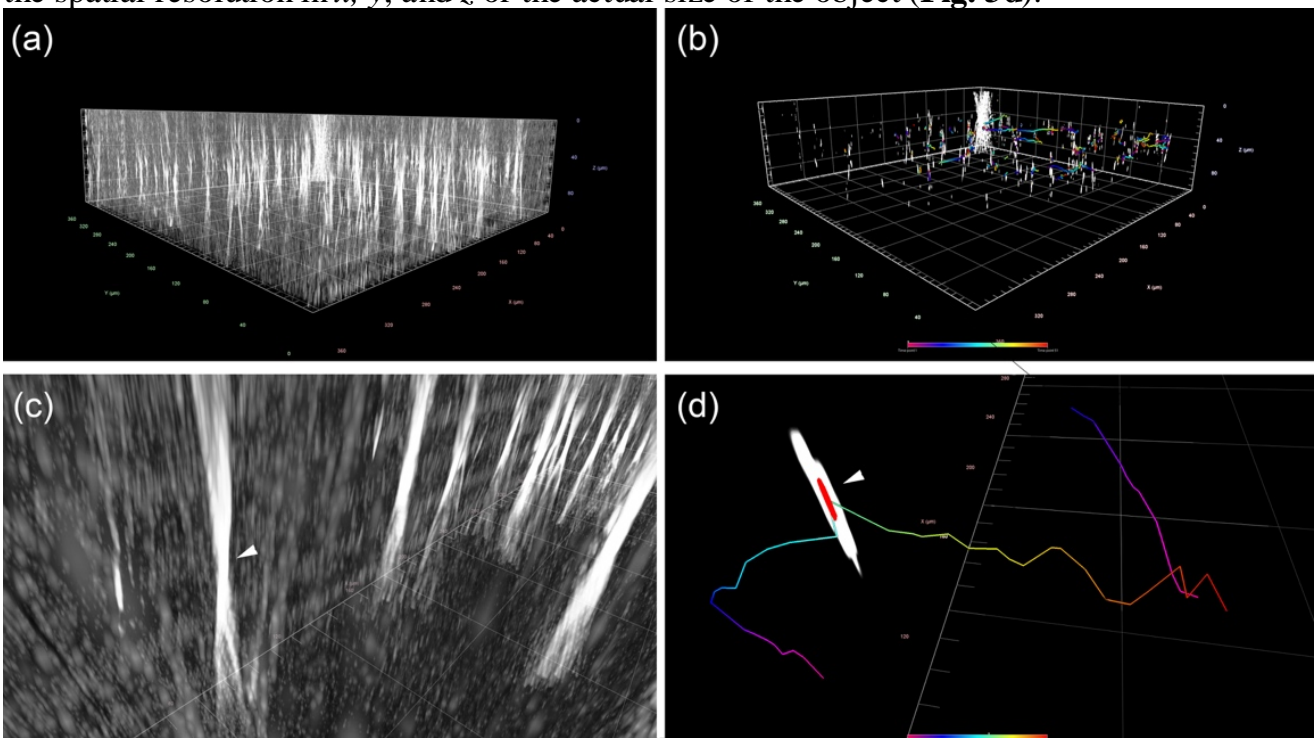

Figure 3. 4D stacks of C. psychrerythraea. (a) 4D phase reconstruction, showing all cells at 51 time points. (b) Phase z-derivative after Gouy processing and tracking (51 time points or 3.4 seconds, 86 tracks total). See Visualization 1 for the full video. (c) Phase reconstruction region of interest (ROI) focused on a single cell (arrow). (d) The same ROI after Gouy processing. Color bar scale reflects the time procession of tracks.

\subsection{Escherichia coli with and without dye staining}

We previously found that a metallocorrole dye increased the phase and amplitude contrast of E. coli cells, permitting thresholding [20]. The goal of this section was to determine the effects of altering the phase contrast of a given cell type on the Gouy phase anomaly. Fig. $\mathbf{4}$ (a, b) show amplitude reconstructions of E. coli without and with dye, and Fig. 4 (c, d) show the corresponding images in phase.

The use of $2 \mu \mathrm{m}$ spacing and the "adhoc" derivative expression (Eq. 4) were essential to cell detection for E. coli. The primary effect of the dye was to increase the $\mathrm{z}$ depth at which the cells were visible (Fig. 5a) as we have previously reported [20]. With or without dye, cells oriented end-on in the field of view showed strong contrast and were easy to detect by any method. However, most cells were oriented lengthwise. These cells showed substantially less contrast, and only the "adhoc" method of addition of the central derivative to the image produced enough contrast to allow thresholding of the derivatives (Fig. $5 \mathbf{b}$, c). 

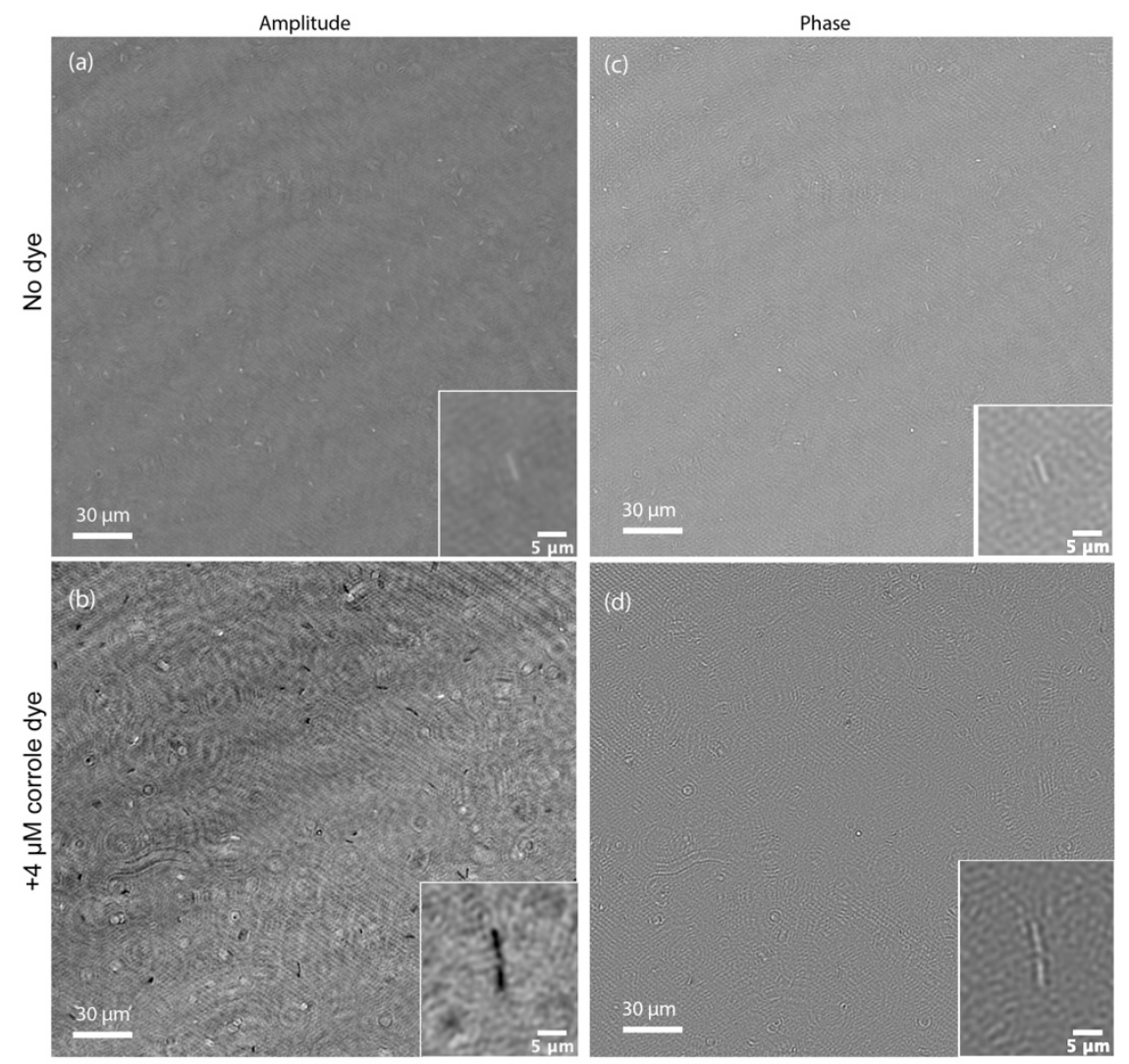

Figure 4. Dye labeling with a dye having a strong absorbance band at the wavelength of the illuminating laser. The full fields of view of the microscope are shown, along with insets of single cells. (a) Unlabeled $E$. coli in amplitude. (b) Dyed E. coli in amplitude. (c) Unlabeled E. coli in phase. (d) Dyed E. coli in phase.

Tracking the undyed E. coli after Gouy processing (Fig. 6) showed similar success to the results achieved with $C$. psychrerythraea. Gouy processing reduced Airy rings and allowed for the localization of cells in $z$. The volume spread function of a single $E$. coli cell reconstructed at $2 \mu \mathrm{m} z$ spacing, as seen in Fig 5c, was reduced to an easily localizable signal after Gouy processing (Fig 5d). Mean \pm SEM height of the volume spread function was $5.46 \pm 0.04 \mu \mathrm{m}, \mathrm{n}$ = 4166 (Supporting Information Table S2).

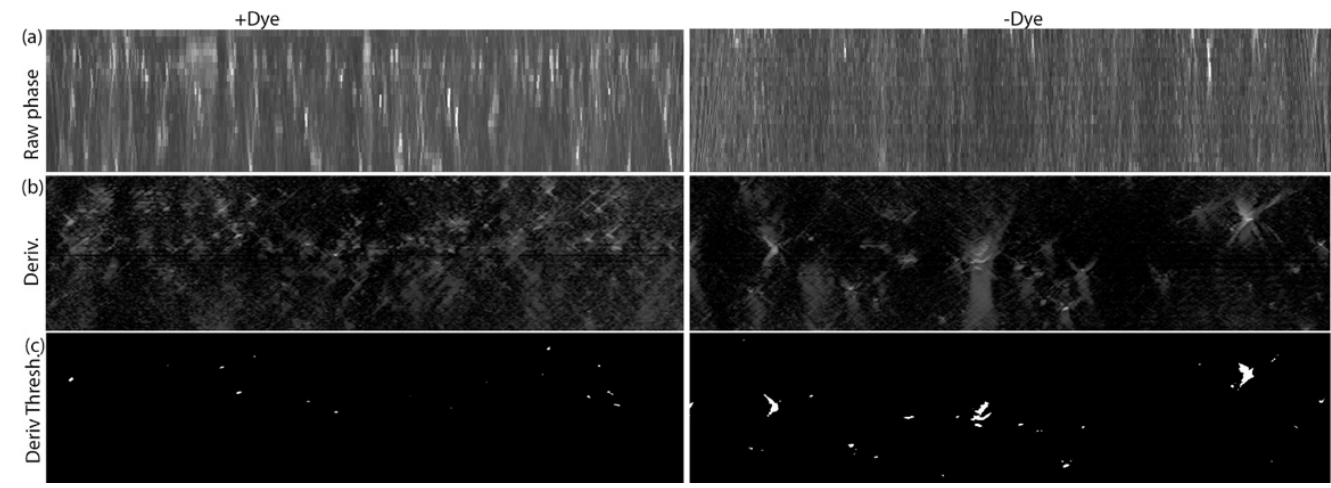

Figure 5. Raw phase, derivatives, and thresholded derivatives of E. coli with and without dye in the $y z$ plane. Panels (a) and (b) are full-scale 32 bit images; panel (c) is binary. (a) In the raw phase images, dyed cells can be seen through more focal planes than unlabeled cells. (b) The derivatives reduce cells in the z plane nearly identically in both cases. (c) Thresholding is possible in both the dyed and undyed case. 

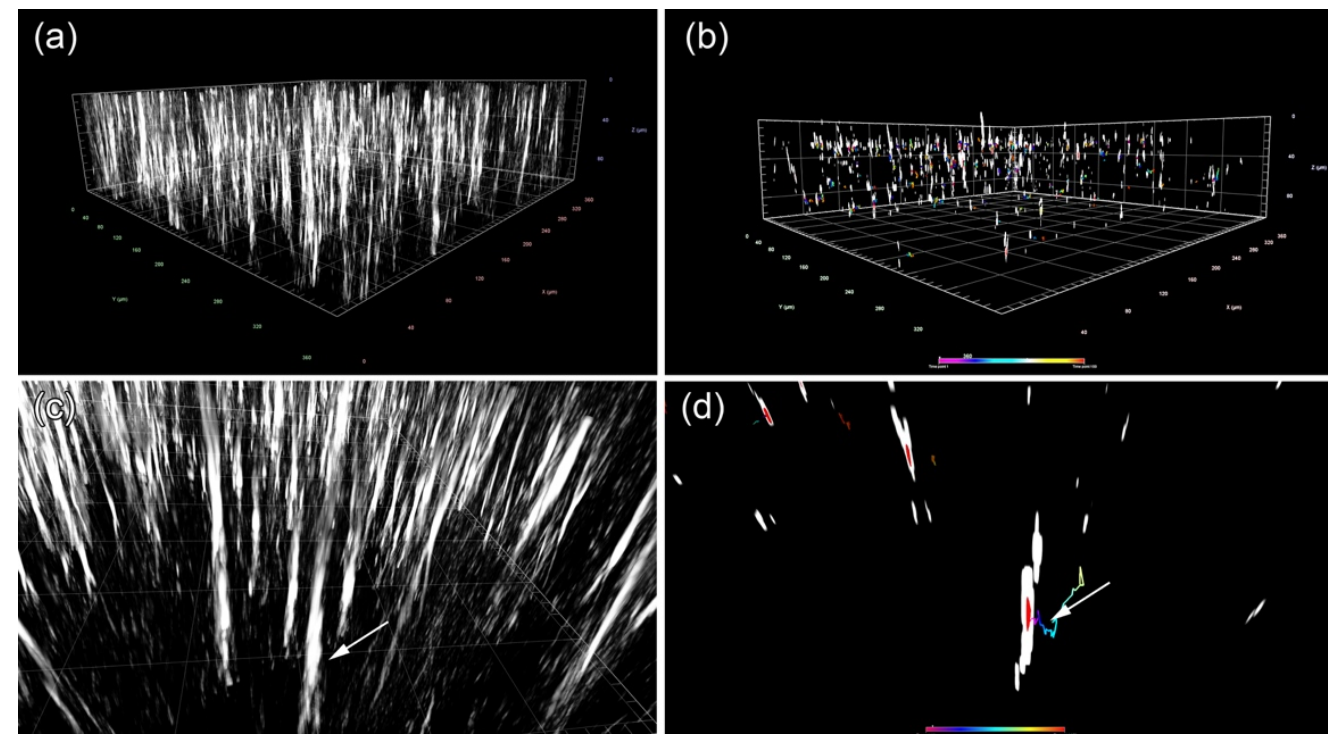

Figure 6. 4D stacks of undyed E. coli. (a) 4D phase reconstruction. Same comments here. As (b) Phase z-derivative after Gouy processing and tracking (100 time points or 6.7 seconds, 127 tracks total). See Visualization 2 for the full video. (c) Phase reconstruction region of interest (ROI) focused on a single cell (arrow). (d) The same ROI after Gouy processing. Color bar scale reflects the time procession of tracks.

In comparison with the undyed $E$. coli, cells dyed with $4 \mu \mathrm{M}$ corrole dye showed enhanced phase signal through multiple $z$ planes. This increased visibility is accompanied by an increase in background signal due to the presence of dye both inside the cell and in the medium , which lessened the contrast gained from the dye (Fig 7a). Nevertheless, Gouy processing of the dyed E. coli still produced results similar to the undyed sample, allowing for robust particle tracking (Fig $\mathbf{7 b}$ ). Volume spread functions of individual cells were minimized by Gouy processing (Fig. 7c, $\mathbf{d}$ just as in the datasets above. The height of the detected particles was somewhat increased compared with undyed cells $(6.27 \pm 0.07 \mu \mathrm{m}, \mathrm{n}=2041)$ (Supporting Information Table S2).

The use of the derivative algorithm for amplitude images reconstructed with OADHM is not useful. For raw amplitude stacks, the derivative images are too noisy to be of use. If holograms are pre-processed by median subtraction prior to reconstruction as described previously, the derivative images are clean but do not yield increased signal to noise over unprocessed stacks (Supporting Information Figure S4). 

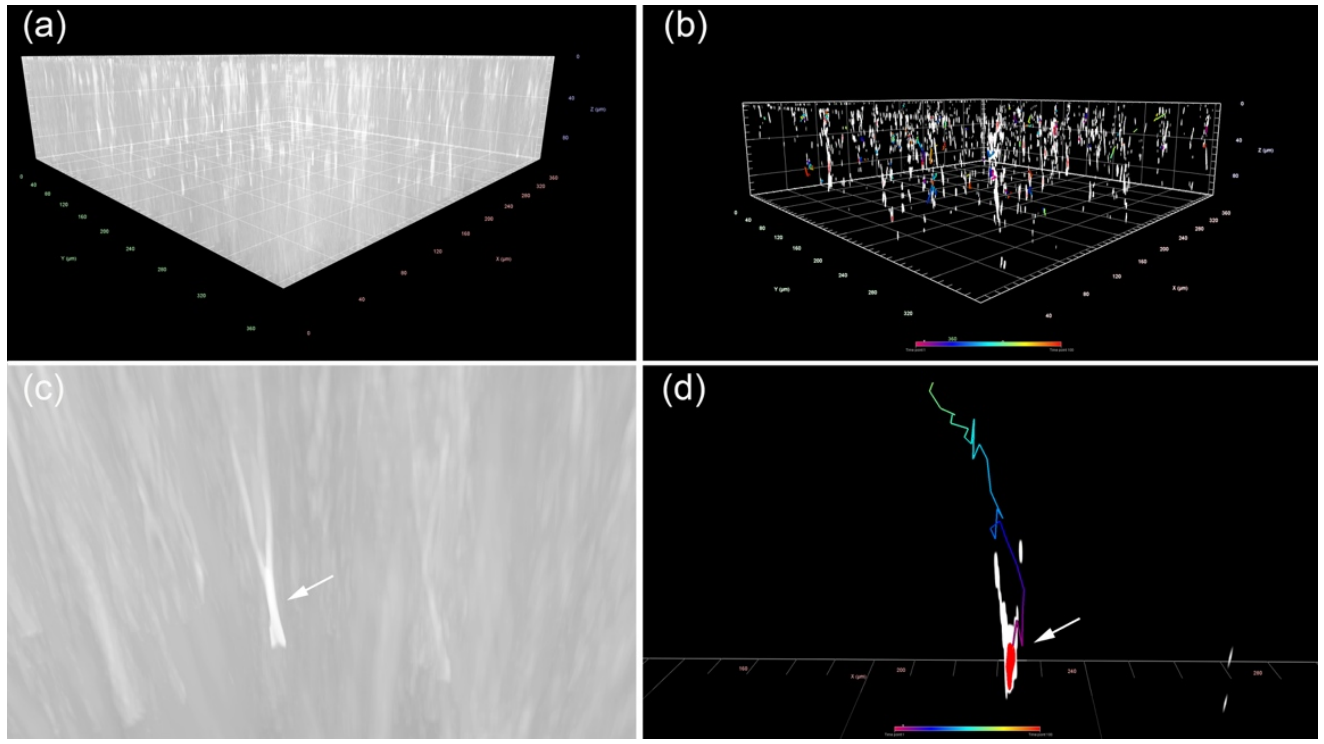

Figure 7. 4D stacks of $E$. coli with $4 \mu \mathrm{M}$ corrole dye. (a) 4D phase reconstruction. Same comment as in Figs. 3 and 6. (b) Phase z-derivative after Gouy processing and tracking (100 time points or 6.7 seconds, 78 tracks total). See Visualization 3 for the full video. (c) Phase reconstruction region of interest (ROI) focused on a single cell (arrow). (d) The same ROI after Gouy processing. Color bar scale reflects the time procession of tracks.

\section{Discussion and Conclusion}

The ability to track small, weak scatterers, such as beads, protein clusters, and bacteria is key to studies of hydrodynamics at low Reynolds number. DHM is a promising technique for instantaneous 3D imaging through relatively large volumes, but methods for processing the resulting amplitude and phase reconstructions are limited. The Gouy phase anomaly provides an effective, computationally inexpensive method for localizing particles in phase volume reconstructions. In all of the test cases shown here, using the Gouy phase anomaly made possible particle tracking in phase reconstructions which would otherwise be difficult to track due to the particles' volume spread functions and other sources of noise. Because our algorithm uses a single pass of pixel-wise arithmetic with only a few operations, it is computationally simple, with $O(N)$ complexity, and requires only a single pass through the image stack. Even very large image stacks ( $>0.5 \mathrm{~Tb}$ or more) may be processed on an ordinary laptop computer in several hours. For example, 2017 Macbook pro 3.1 GHz Intel Core i7, 16 GB RAM, solid state drive, running FIJI/ImageJ2.00-rc-69/1.52u, 8 bit slices, the algorithm required 30 $\mathrm{ms} / \mathrm{slice}$ for 8-bit images. For 32 bit images it was $0.89 \mathrm{~s} / \mathrm{slice}$ for 200 slice depth and twice that for 401 slice depth (timing performed using the timing features available in imageJ macros). All timing includes disk and virtual memory I/O operations.

Other commonly used methods for partical localization are more computationally intensive or constrained in applicability. Deconvolution is frequently used to localize particles in the $\mathrm{z}$ plane, but the volume-spread functions produced by DHM are very different than those seen in fluorescence microscopy, so commercially available deconvolution software is inadequate. A few papers on holographic deconvolution are available [27] [28] but deconvolution is computationally expensive, with complexity $O(N \log N)$ except for special cases. Fitting the Airy ring patterns of raw holograms to scattering models, can be extremely precise but is mostly of use when the size and refractive index of the particles are known in advance and when the particles can be treated using Mie theory [29]. More complex models using the discrete dipole approximation have been published but are at least $O(N \log N)$ or $O\left(N^{2}\right)$ and depend on 
convergence criteria [30, 31]. Fitting of intensity distributions to Gaussian functions has also been demonstrated with particles including bacterial cells [32], but even for linear fits requires greater computation than our approach, which requires only a few arithmetic operations at each pixel.

Phase images of bacterial cells can be more useful than amplitude images for particle localization and tracking because of the absence of speckle noise. Some bacterial strains show stronger phase contrast than others, a field which remains entirely unexplored. One of our test strains in this study, Collwellia psychrerythraea $34 \mathrm{H}$, shows excellent phase contrast. This may be related to the fact that it is surrounded by a capsular polysaccharide structure (CPS) which affects the scattering properties of the cells, acting as a "photon trap" [33]. Growth under conditions that alter CPS expression may influence phase contrast. Phase imaging may be useful for identifying conditions and bacterial strains that produce a CPS. Other physiological parameters may also influence phase contrast; increased attention to the utility of phase imaging in microbiology may lead to surprising findings. For practical purposes, some datasets are more readily tracked in amplitude images, and others in phase. Cell-independent factors such as noise in the specific experimental run, sample chamber composition and cleanliness, and the presence of artifacts can all affect quality of the amplitude and phase images differently. Having tools for processing both types of images increases the chances of successful cell tracking.

An interesting finding here is that dye labeling does not produce cells with greater phase shifts in any given $z$ plane, but instead less attenuation of contrast with depth than in undyed cells. This is of little use when using the Gouy phase method, and also suggests that the source of contrast in cells like Colwellia is not merely a greater refractive index of the cell wall. Further experimentation with other dyes and other contrast agents is needed to create generally useful labeling techniques for phase imaging.

The algorithm for differentiation is given in the Supporting Information and may be easily integrated with our FIJI reconstruction package or implemented in other platforms. Differentiation algorithms with smoothing are also readily available in many other software packages. This technique is readily accessible to anyone working with volumetric phase images. All raw datasets are available from the authors upon request.

\section{Funding, acknowledgments, and disclosures}

\subsection{Funding}

National Science Foundation (NSF) 1828793. Portions of this work were supported under a contract from or performed at the Jet Propulsion Laboratory, California Institute of Technology, under a contract with the National Aeronautics and Space Administration.

\subsection{Acknowledgments}

We thank J. Kent Wallace and Louis Sumrall for useful discussions. The FeatureJ plug-ins were written by Erik Meijering.

\subsection{Disclosures}

The authors declare no conflicts of interest.

See Supplement 1 for supporting content.

\section{References}

1. L. G. Gouy, "Sur une propriété nouvelle des ondes lumineuses," C. R. Acad. Sci. Paris 110(1890).

2. $\quad$ L. G. Gouy, "Sur la propagation anormale des ondes,"C. R. Acad. Sci. Paris 111(1890).

3. P. Debye, "Das Verhalten von Lichtwellen in der Nähe eines Brennpunktes oder einer Brennlinie," Ann Phys-Berlin 335, 755-776 (1909). 
4. A. Rubinowicz, "On the anomalous propagation of phase in the focus," Phys Rev 54, 931-936 (1938).

5. E. H. Linfoot and E. Wolf, "PHASE DISTRIBUTION NEAR FOCUS IN AN ABERRATION-FREE DIFFRACTION IMAGE," Proceedings of the Physical Society of London Section B 69, 823-832 (1956).

6. M. S. Kim, T. Scharf, C. Rockstuhl, and H. P. Herzig, "Phase Anomalies in Micro-Optics," in Progress in Optics, Vol 58, E. Wolf, ed. (2013), pp. 115-197.

7. P. Baudoz, Y. Rabbia, and J. Gay, "Achromatic interfere coronagraphy - I. Theoretical capabilities for ground-based observations," Astronomy \& Astrophysics Supplement Series 141, 319-329 (2000).

8. P. Baudoz, Y. Rabbia, J. Gay, R. Burg, L. Petro, P. Bely, B. Fleury, P. Y. Madec, and F. Charbonnier, "Achromatic interfere coronagraphy II. Effective performance on the sky," Astronomy \& Astrophysics Supplement Series 145, 341-350 (2000).

9. C. B. Giuliano, R. Zhang, and L. G. Wilson, "Digital inline holographic microscopy (DIHM) of weaklyscattering subjects," J Vis Exp, e50488 (2014).

10. L. G. Wilson, L. M. Carter, and S. E. Reece, "High-speed holographic microscopy of malaria parasites reveals ambidextrous flagellar waveforms," Proc Natl Acad Sci U S A 110, 18769-18774 (2013).

11. K. L. Thornton, J. K. Butler, S. J. Davis, B. K. Baxter, and L. G. Wilson, "Haloarchaea swim slowly for optimal chemotactic efficiency in low nutrient environments, " Nat Commun 11, 4453 (2020).

12. U. Schnars and W. P. O. Juptner, "Digital recording and numerical reconstruction of holograms, "Meas Sci Technol 13, R85-R101 (2002).

13. G. Nehmetallah, L. Williams, T. Nguyen, H. Li, and S. Matthews, "Latest advances in single and multiwavelength digital holography and holographic microscopy. ," Asian J Phys. 25, 609-630 (2016).

14. Y. Park, C. Depeursinge, and G. Popescu, "Quantitative phase imaging in biomedicine," Nat Photonics 12, 578-589 (2018).

15. Y. Liu, Z. Wang, and J. H. Huang, "Recent Progress on Aberration Compensation and Coherent Noise Suppression in Digital Holography," Appl Sci-Basel 8(2018).

16. P. Y. Liu, L. K. Chin, W. Ser, T. C. Ayi, P. H. Yap, T. Bourouina, and Y. Leprince-Wang, "Real-Time Measurement of Single Bacterium's Refractive Index using Optofluidic Immersion Refractometry," in 28th European Conference on Solid-State Transducers, G. Sberveglieri and V. Ferrari, eds. (2014), pp. 356-359.

17. J. J. Koenderink and A. J. van Doorn, "Representation of local geometry in the visual system," Biol Cybern 55, 367-375 (1987).

$18 . \quad$ J. K. Wallace, S. Rider, E. Serabyn, J. Kuhn, K. Liewer, J. Deming, G. Showalter, C. Lindensmith, and J. Nadeau, "Robust, compact implementation of an off-axis digital holographic microscope," Opt Express 23, 17367-17378 (2015).

19. C. A. Lindensmith, S. Rider, M. Bedrossian, J. K. Wallace, E. Serabyn, G. M. Showalter, J. W. Deming, and J. L. Nadeau, "A Submersible, Off-Axis Holographic Microscope for Detection of Microbial Motility and Morphology in Aqueous and Icy Environments," Plos One 11(2016).

$20 . \quad$ J. L. Nadeau, Y. B. Cho, and C. A. Lindensmith, "Use of dyes to increase phase contrast for biological holographic microscopy," Opt Lett 40, 4114-4117 (2015).

21. J. Schindelin, I. Arganda-Carreras, E. Frise, V. Kaynig, M. Longair, T. Pietzsch, S. Preibisch, C. Rueden, S. Saalfeld, B. Schmid, J. Y. Tinevez, D. J. White, V. Hartenstein, K. Eliceiri, P. Tomancak, and A. Cardona, "Fiji: an open-source platform for biological-image analysis," Nat Methods 9, 676-682 (2012).

22. D. Cohoe, I. Hanczarek, J. K. Wallace, and J. Nadeau, "Multiwavelength imaging and unwrapping of protozoa in amplitude and phase using custom Fiji plug-ins," Frontiers in Physics 7(2019).

23. C. Mann, L. Yu, C. M. Lo, and M. Kim, "High-resolution quantitative phase-contrast microscopy by digital holography," Opt Express 13, 8693-8698 (2005).

24. T. Colomb, J. Kuhn, F. Charriere, C. Depeursinge, P. Marquet, and N. Aspert, "Total aberrations compensation in digital holographic microscopy with a reference conjugated hologram," Opt Express 14, 4300-4306 (2006).

25. T. Colomb, E. Cuche, F. Charriere, J. Kuhn, N. Aspert, F. Montfort, P. Marquet, and C. Depeursinge, "Automatic procedure for aberration compensation in digital holographic microscopy and applications to specimen shape compensation," Appl Opt 45, 851-863 (2006).

26. M. Bedrossian, J. K. Wallace, E. Serabyn, C. Lindensmith, and J. Nadeau, "Enhancing final image contrast in off-axis digital holography using residual fringes," Opt Express 28, 16764-16771 (2020).

$27 . \quad$ L. Dixon, F. C. Cheong, and D. G. Grier, "Holographic deconvolution microscopy for high-resolution particle tracking," Opt. Express 19, 16410-16417 (2011).

28. T. Latychevskaia and H. W. Fink, "Holographic time-resolved particle tracking by means of threedimensional volumetric deconvolution," Opt Express 22, 20994-21003 (2014).

$29 . \quad$ S. H. Lee, Y. Roichman, G. R. Yi, S. H. Kim, S. M. Yang, A. van Blaaderen, P. van Oostrum, and D. G. Grier, "Characterizing and tracking single colloidal particles with video holographic microscopy," Opt Express 15, 18275-18282 (2007).

30. A. Wang, R. F. Garmann, and V. N. Manoharan, "Tracking E. coli runs and tumbles with scattering solutions and digital holographic microscopy," Opt Express 24, 23719-23725 (2016).

31. B. T. Draine and P. J. Flatau, "Discrete-Dipole Approximation for Scattering Calculations," J Opt Soc Am A 11, 1491-1499 (1994). 
32. G. Huang, W. Tian, M. Qi, X. Gong, and G. Zhang, "Improving axial resolution for holographic tracking of colloids and bacteria over a wide depth of field by optimizing different factors," Opt Express 26, 99209930 (2018).

33. A. W. Decho and T. Gutierrez, "Microbial Extracellular Polymeric Substances (EPSs) in Ocean Systems," Front Microbiol 8, 922 (2017). 